\title{
EVALUACIÓN DE IMPACTO AMBIENTAL: FUTURA LÍNEA DE ALTA TENSIÓN (LAT) 132KV, VINCULO: DESDE ET500KV PASO DE LA PATRIA A FUTURA ET PIRAYÚ Y DESDE ET PIRAYÚ HASTA ITÁ IBATÉ, PROVINCIA DE CORRIENTES
}

Magister Ingeniero Emilio Fabián Scozzina. Dr. Ciencias Biológicas José Luis Fontana. Magister Ingeniero Agrónomo Héctor Currie. Dra. María Eugenia Senosiain Verrastro. $\left(^{*}\right)$

Palabras Claves: Líneas eléctricas, alta tensión, evaluación de impacto ambiental.

\section{INTRODUCCIÓN:}

El uso de energía eléctrica en la sociedad moderna está asociado a la generación bienes y servicios que redundan en una mejora de la calidad de vida de los habitantes.

Históricamente energía eléctrica se transmite desde los distintos puntos de generación a los de consumo, mediante redes eléctricas. Esta es la forma más rentable para su distribución entre los distintos centros urbanos, como es el caso las localidades de Paso de la Patria e Itá Ibaté, junto a otras localidades intermedias, ubicadas al norte de la provincia de Corrientes, república Argentina, donde se construirán los vínculos, objeto del presente trabajo.

El Proyecto de ingeniería e interconexión fue desarrollado por la Secretaria de Energía de la Provincia de Corrientes y beneficia directamente a toda la zona norte de la provincia, también posibilitara saldar una deuda histórica en materia de infraestructura eléctrica. El estudio de Impacto Ambiental (EIA) fue solicitado por esa Secretaria al Departamento de Ingeniería de la Facultad de Ciencias Exactas Naturales y Agrimensura de la UNNE.

La preocupación por el cuidado del medio ambiente no escapa a las obras de infraestructura eléctrica destinadas a la transmisión de energía, en este caso las líneas de alta tensión (LAT). La Evaluación de Impacto Ambiental (EIA), es considerada una herramienta de gestión para la protección del medio ambiente, la debe ser presentada a la autoridad provincial competente, el ICAA.

La Facultad de Ciencias Exactas Naturales y Agrimensura, según la Resolución No655/14 de del Consejo Directivo, creó el "Grupo de Investigación en Gestión Ambiental de Redes Eléctricas", para así poder atender las demandas y prestar servicios en la EIA del presente proyecto. Siendo que la

(*) Grupo de Investigación en Gestión Ambiental de Redes Eléctricas de la Facultad de Ciencias Exactas de la UNNE - efscozzina@gmail.com 
naturaleza del estudio es multidisciplinaria y transversal a distintas áreas se creó un equipo de trabajo Ad Doc, conformado por distintos profesionales expertos en la materia.

El objetivo de las tareas a realizar alcanza a las siguientes actividades: EIA del Tramo No1 LAT132 KV desde la Estación Transformadora ET $500 \mathrm{KV}$ Paso de La Patria, hasta futura ET Pirayú. EIA Tramo No2 LAT $132 \mathrm{KV}$, desde futura ET Pirayú hasta la localidad de Itá Baté, provincia de Corrientes.

\section{METODOLOGÍA UTILIZADA:}

La metodología aplicada para la elaboración de la EIA de la Líneas de Alta Tension (LAT), resulta de la combinaciones de valoraciones cualitativas, basado en el método de causa y efecto utilizado en la Matriz de Leopold. Al que se adiciona en el caso de las valoraciones cuantitativas en la identificación de impactos, el Método BatelleColumbus. Estos son verdaderos clásicos, adaptables para en la determinación sistemática de impactos ambientales en distintos tipos de proyectos.

\begin{tabular}{|l|l|}
\hline Método & Características \\
\hline Batelle-Columbus & $\begin{array}{l}\text { Método cuantitativo desarrollado para la evaluación de } \\
\text { impactos ambientales. Permite la evaluación sistemática de } \\
\text { los impactos de un proyecto mediante el empleo de } \\
\text { indicadores homogéneos.Es fácilmente modificable a otros } \\
\text { proyectos. Fue desarrollado en los Laboratorios de Batalle- } \\
\text { Columbus, en 1972. }\end{array}$ \\
\hline $\begin{array}{l}\text { Matriz de causa efecto } \\
\text {-Leopold }\end{array}$ & $\begin{array}{l}\text { Método cualitativo de evaluación de impacto ambiental que } \\
\text { fue creado en 1971. }\end{array}$ \\
\hline
\end{tabular}

\section{ESTADO DE AVANCE DEL ESTUDIO.}

El presente estudio comprende un análisis pormenorizado de impactos en el medio ambiente, donde se desarrolla el proyecto, este se encuentra caracterizado por:

El Medio Físico y sus sub sistemas: 1Medio Inerte: aire, tierra, agua. 2- Medio Biótico: flora y fauna. 3- Perceptual: unidades de paisaje. Por último el Medio Socio-económico: constituido por aspectos sociales, legales, (histórico-culturales-patrimoniales) y económicos de la población en los departamentos afectados.

El estudio de EIA se encentra en estado avanzado y próximo a su presentación ante la autoridad competente provincial, el ICAA. En el Tramo No1 ET 500 Kv (ET Paso de la Patria) hasta futura ET Pirayú (Localidad de Paso de la Patria). Se evalúa el impacto ambiental de una línea en de $132 \mathrm{KV}$ con disposición coplanar vertical, y estructura para montar un segunda terna, con postración de hormigón armado. Este tramo de línea cubre una extensión 16.289 metros y área impactada de 495.185,6 m2. $\mathrm{El}$ ancho de la franja del electroducto aprobado es de 30,40 metros.

El segundo tramo de traza arranca en la futura ET $132 \mathrm{Kv}$ ET Pirayú ubicada en Paso de la Patria hasta la ET Itá Iba- 


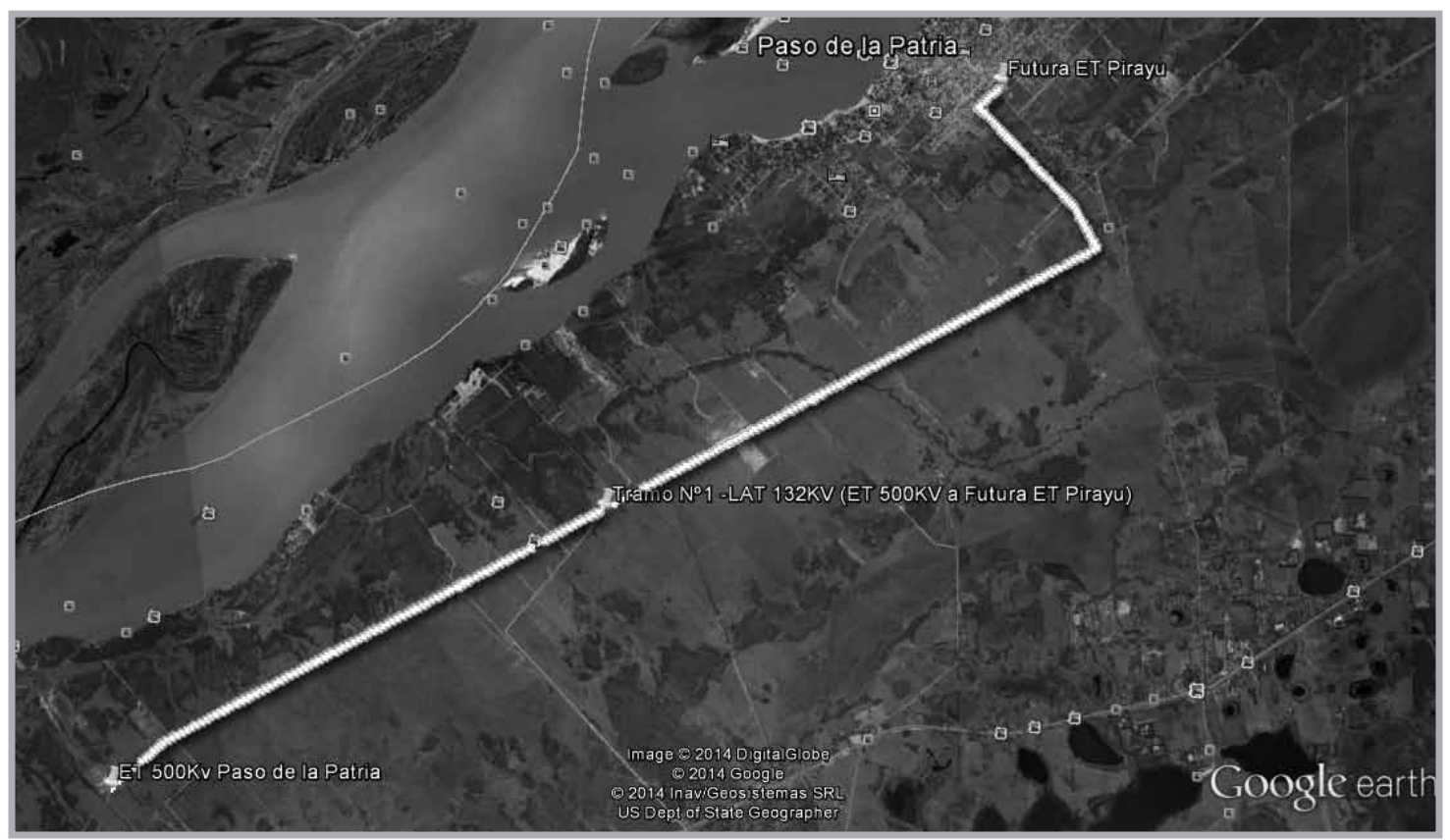

Figura No1 Se muestra la traza de la LAT 132KV en su primer tramo.

té. El futuro tramo de línea de LAT 132 KV cubre una extensión 128.762 metros y área impactada aproximadamente es de $4.506 .670 \mathrm{~m} 2$. El ancho de la franja estimada de servidumbre para el electroducto es de 35 metros.

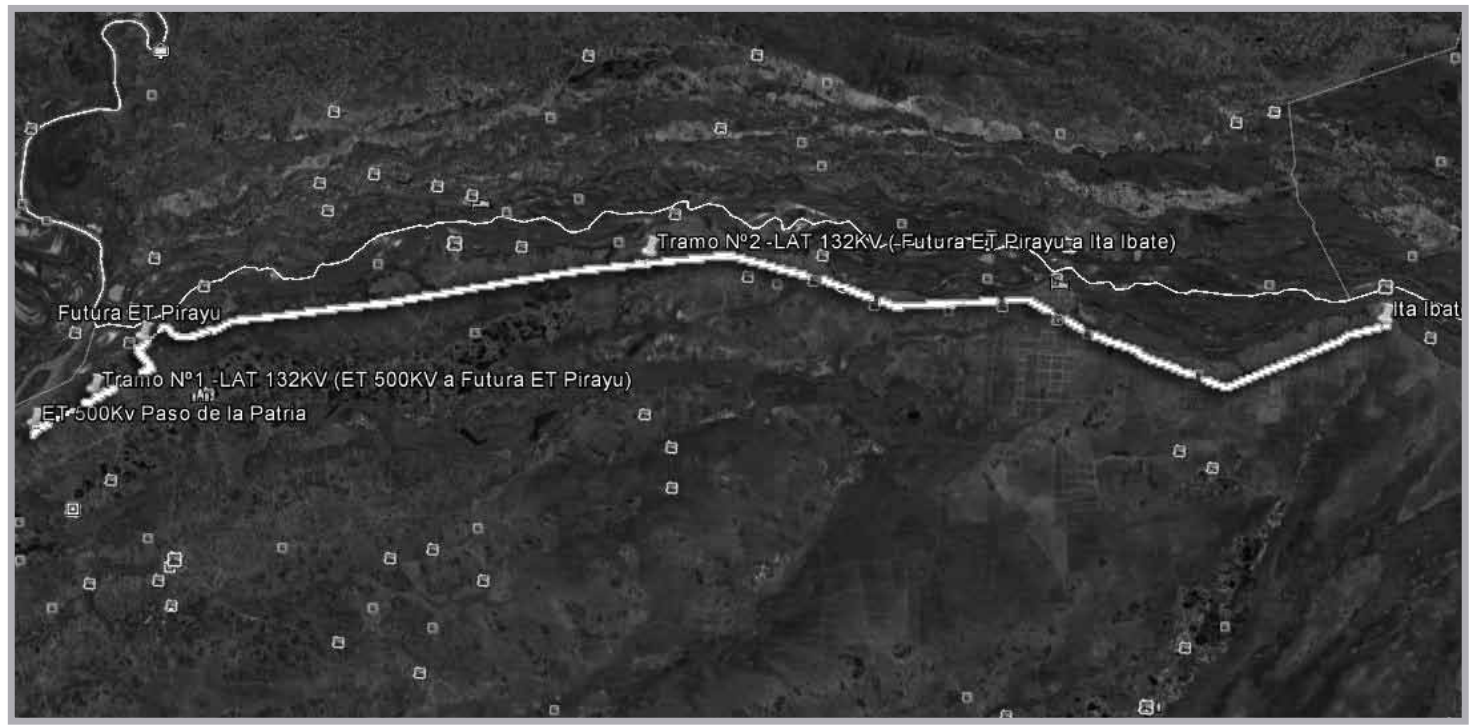

Figura No2 Se muestra la traza de la LAT 132KV en su segundo tramo. 


\section{RECOMENDACIONES PRE- LIMINARES.}

$\mathrm{Si}$ bien los estudios se encuentran en etapa avanzada de elaboración, se pueden adelantar algunas de las recomendaciones consideradas desde punto de vista ambiental, en gestión de las redes eléctricas.

$\checkmark$ Un desbroce inicial (desmonte y desmalezado) selectivo mediante utilización de medios mecánicos (desmalezadora) o manuales (hacha, machete).

$\checkmark \quad$ La utilización de herbicidas para mantener libre el electroducto no es aconsejable no sólo porque no son selectivos, sino por su posible dispersión a cuerpos de agua cercano (cañadas, arroyos), contaminación de napa freática, cultivos en pequeña escala.

$\checkmark \quad$ Frente a la necesaria construcción de caminos, se sugiere evitar el efecto "barrera" y represa de aguas en zonas deprimidas, con obras que permitan el normal escurrimiento.

$\square \quad$ Se sugiere dejar avanzar el proceso natural de revegetación de los caminos de servicio, manteniéndolos sólo con desmalezado periódico. Esto significará un menor costo de mantenimiento al evitarse los procesos erosivos, a la vez de un menor impacto biológico y visual.

$\checkmark$ De ser posible, el acceso a la traza a partir del camino de servidumbre de la LAT $500 \mathrm{KV}$, ayudaría a reducir considerablemente los impactos negativos.

$\checkmark \quad$ No trabajar en la zona correspondiente al electro ducto de la LAT de 500 $\mathrm{KV}$, lindero a la construcción de la nueva LAT $132 \mathrm{KV}$. $\square \quad$ Para el caso las operaciones requiera extracción de suelo de préstamo, se propone reutilizar, las extracciones hechas para la fundaciones de las columnas.

$\checkmark \quad$ Radiaciones no ionizantes: Se recomienda la observancia rigurosa de las normas de seguridad, provinciales, nacionales o internacionales existentes y en particular de las OMS.

$\checkmark \quad$ El nivel Isoceráunico de un lugar puede ser afectado por la presencia de la LAT, ya que se modifican las alturas naturales del terreno.

$\square \quad$ Presencia de potenciales peligrosos por las puestas a tierra.

$\checkmark \quad$ Se recomienda tener un plan de manejo y control de fuegos en caso de incendios e implementar las medidas de prevención correspondientes con de coordinación con bomberos de las localidades.

$\square$ Evitar que los hoyos de fundación estén al descubierto en su parte superior ya que resulta un peligro para especies animales que pueden caer dentro de ellos.

\section{CONCLUSIONES PRELIMI- NARES.}

Se presentan las conclusiones para la LAT $132 \mathrm{KV}$ en su primer tramo, ya que resulta esta la más avanzada en la etapas de obras, según requerimientos. En posteriores publicaciones se publicara un informe completo de toda la traza, hasta la localidad de Itá Ibaté.

La nueva línea aporta a la mejora del desarrollo local sustentable, y reemplaza a una vieja línea que se encuentra sobre la 
vera Ruta Nacional 12 que tiene cerca de 50 años de antigüedad (En el caso de $\mathrm{Co}^{-}$ rrientes- Paso de la Patria). Constituye una infraestructura imprescindible y básica para permitir el desarrollo y evitar el desarraigo, en una zona de creciente actividad económica como lo es la ciudad de Paso de la $\mathrm{Pa}-$ tria.

Socioeconómicamente ayuda al dinamismo comercial y la prestación de servicios; Además de distender las situaciones de conflicto con los usuarios, que se dan con picos consumo en verano e invierno. La situación más crítica se sucede en verano, cuando la villa turística está a pleno, y se producen cortes suministro eléctrico por fallas o picos en la demanda.

Desde el punto de vista de la sustentabilidad podemos decir que la provincia de $\mathrm{Co}^{-}$ rrientes aporto durante el 2013 con 17.178 GWh de energía generados hidroeléctricamente. Si comparamos la energía eléctrica generada, por todas las centrales hidroeléctricas de la República Argentina durante el año 2013, esto representó el 31,1\% del total nacional. Siguiendo este razonamiento Yacyretá aportó el 44,03\% de esa energía hidroeléctrica, la que procede de una fuente renovable. Es decir un 13,69 del total nacional.

Resulta una contradicción que con tal nivel de generación, partiendo de un recurso renovable, no existan infraestructuras adecuadas para su distribución, lo cual justifica la implementación de estas LAT, siguiendo los cuidados ambientales necesarios y minimizando los impactos, de manera de hacerlos tolerables.
Existe una asimetría importante ya que para cubrir la demanda creciente ENARSA, con el "Plan Generación Distribuida I", desplego generación distribuida con grupos de generadores diésel 5MW, en la localidad de Paso de la Patria Estos equipos entraron en servicio 28/05/2009.

La asimetría se da, ya que al tener una fuente de energía renovable como la hidroeléctrica, se genera energía eléctrica mediante el quemado de combustibles fósiles. Citamos como ejemplo que el 26/06/2014 la generación diésel de Paso de la Patria aporto 4MW (Fuente CAMMESA).

No se pretende abrir juicio sobre las situaciones o estrategias empleadas para satisfacer en tiempo y forma, la demanda de energía eléctrica, simplemente resaltar el hecho de que existe una generación en diésel, que puede ser reemplazada o dejarla como reserva fría ante cualquier eventualidad. Evitando las consiguientes emisiones de $\mathrm{CO} 2$.

\section{EQUIPO INVESTIGADOR.}

Los autores del presente artículo forman parte del Grupo de Investigación en Gestión Ambiental de Redes Eléctricas. Director Magister Ingeniero Scozzina, Emilio Fabián, Dr. Ciencias Biológicas José Luis Fontana de la Facultad de Ciencias Exactas UNNE. Magister Ingeniero Agrónomo Héctor Currie, de la Facultad de Ciencias Agrarias de la UNNE. Dra. María Eugenia Senosiain Verrastro de la Facultad de Abogacía de la UNNE. Contacto efscozzina@gmail.com - Tel. Celular:0362-154527366. 